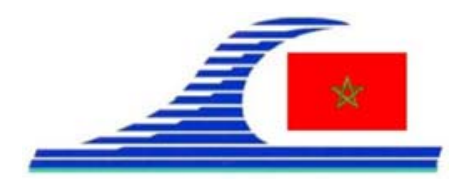

Conférence Méditerranéenne Côtière et Maritime

EDITION 2, TANGER, MAROC (2011)

Coastal and Maritime Mediterranean Conference

Disponible en ligne - http://www.paralia.fr - Available online

\title{
Port Tanger Méditerranée : Etude géologique et géotechnique, et analyse du risque de liquéfaction à partir des essais in situ
}

\author{
Mohammed Reda AOULAD MANSOUR ${ }^{1,3}$, Abdelilah MIHRAJE ${ }^{2}$, \\ Jamal Eddine STITOU MESSARI ${ }^{3}$
}

1. Agence Spéciale Tanger Méditerranée (TMSA), ZFE de Ksar El Majaz, Oued Rmel Route de Fnideq, Maroc.

aouladmansour@gmail.com

2. Direction de l'Aménagement du Port Tanger Méditerranée (DAPTM), ZFE de Ksar El Majaz Oued Rmel Route de Fnideq, Maroc. amihraje@yahoo.fr

3. Université Abdelmalek Essaadi, Faculté des Sciences de Tétouan, BP 2121 Tétouan, Maroc.jstitou@uae.ma

\section{Résumé :}

Le site du Port Tanger Méditerranée, situé sur les flyschs de Tisirène de l'unité de Tanger faisant partie du domaine externe rifain, a fait l'objet de plusieurs campagnes de reconnaissances géotechniques qui ont permis d'établir une synthèse géologique et géotechnique et de repérer des couches de sables saturés susceptibles de liquéfier sous l'effet des séismes. L'analyse du risque de liquéfaction est conduite sur la base des essais in situ (CPTU) et selon les deux séismes de projets. Elle montre que la zone à risque est réduite et se situe entre les CPT22 et 25 et que le séisme lointain est plus néfaste pour le terrain que le séisme de source proche. La charge appliquée par l'ouvrage réduit ou atténue selon les cas le potentiel de liquéfaction et seule subsiste la partie superficielle du sol d'environ 3m de hauteur.

\section{Mots-clés :}

Port Tanger Med - Géologie - Géotechnique - Essais in situ - Séisme de projet Risque de liquéfaction

\section{Introduction}

Situé entre les villes de Ksar Sghir et Fnideq à l'extrémité Nord occidentale du Maroc, le site du port Tanger Med est traversé par l'embouchure d'Oued Rmel. Les assises géologiques sont des alternances de grés et de pélites du domaine des flyschs rifains, les bancs métriques gréseux dominent sur la rive droite du port, tandis que sur la rive gauche dominent les pélites. Ce travail consiste en :

- Une synthèse géologique et géotechnique des données des différentes campagnes de reconnaissance réalisées dans la zone du port ;

- Une étude du risque de liquéfaction des sols sableux à partir des essais in situ. 
La connaissance de la Mer :

un vecteur du développement durable en Méditerranée

\section{Etude géologique et géotechnique}

\subsection{Campagnes géotechniques et sondages réalisés}

Le site du port de Tanger méditerranée a fait l'objet de plusieurs campagnes de reconnaissances échelonnées dans le temps et conduites par plusieurs laboratoires (LPEE, FUGRO, SEACORE, GTEC). Ainsi plusieurs sondages et essais ont été réalisés. L’ensemble des données est utilisé dans cette étude.

\subsection{Réalisation de la carte du substratum}

Les données des différentes campagnes traitées par les logiciels SURFER et COVADIS ont permis de tracer une carte du substratum et le bloc diagramme correspondant (figure 1). Ces derniers montrent la présence de 3 vallées correspondant à Oued Rmel, Oued Chaâba et un petit cours d'eau.

Il est à signaler également que le substratum plonge brutalement vers le large. La superposition de la carte avec le plan de masse du port (figure 1) montre que les 3 vallées traversent les terre-pleins, l'emplacement du quai à conteneurs et certaines se prolongent même sous l'emprise des ouvrages de protection.
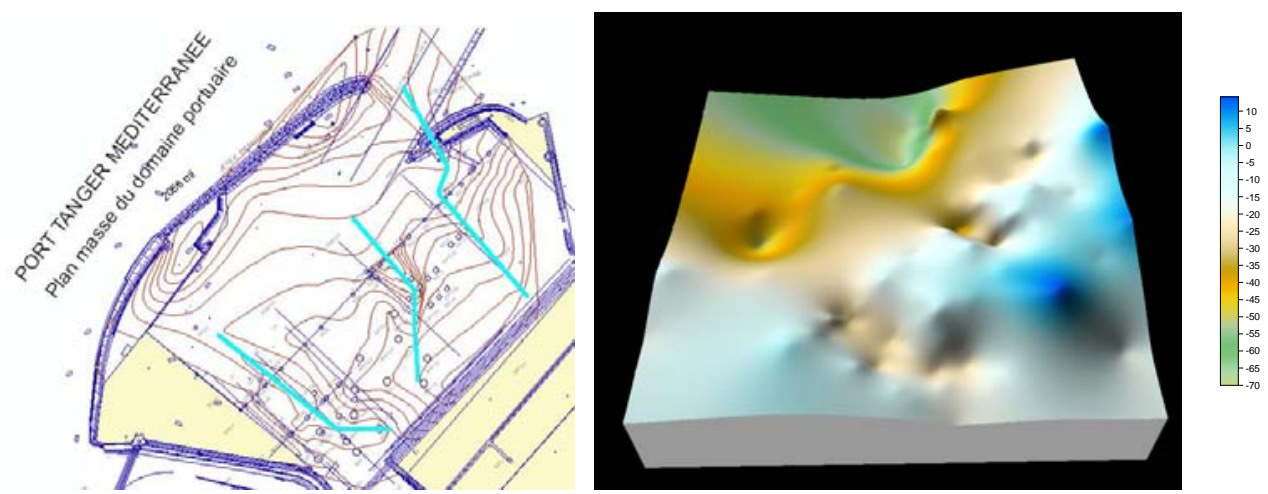

Figure 1. Carte et bloc diagramme du substratum réalisée sous Surfer.

\subsection{Réalisation des coupes géologiques}

Trois coupes géologiques ont été établies à l'emplacement des principaux ouvrages du port (figure 2). Elles montrent l'existence d'une épaisse couche de sables et sables silteux à l'emprise des ouvrages de protection. Ces formations, saturées d'eau, risquent de liquéfier sous l’effet des séismes. Il convient alors d’évaluer ce risque. 


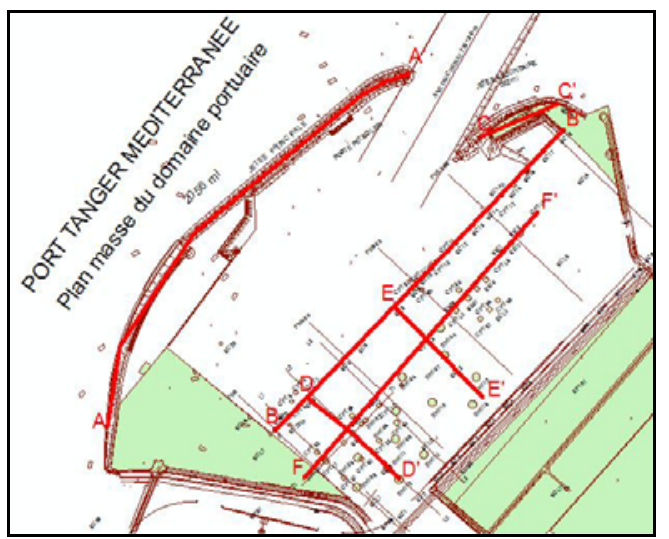

Figure 2. Localisation des essais et des coupes par rapport aux ouvrages du port.

\section{Analyse du risque de liquéfaction}

\subsection{Etude de l'aléa sismique}

Cette étude est réalisée d'abord selon une approche déterministe (CHERKAOUI, 2003) puis par une approche probabiliste (GEOTER International, 2004). Elle a permis de retenir deux séismes de projet, un pour la source proche et un pour la source lointaine dont les caractéristiques de magnitude, d'accélération sont 4,7 et 0,24 g pour la source proche et 8,5 et 0,093 $\mathrm{g}$ la source lointaine. La période de retour est respectivement 1975 et 475 ans. L’étude de la liquéfaction est entamée sur la base de ces résultats.

\subsection{Les essais in situ}

L’analyse du risque de liquéfaction est conduite notamment à partir des CPTU.

\subsection{L’analyse du risque de liquéfaction et le calcul des facteurs de sécurité}

Elle est réalisée selon la Méthode empirique de YOUD et IDRISS (2001) qui consiste à vérifier d'abord si les sols en place sont potentiellement liquéfiables, puis procéder à une étude préliminaire du risque sans charge de l'ouvrage et enfin évaluer l'influence du chargement apporté par l’ouvrage.

L'évaluation du risque de liquéfaction se base sur le calcul d'un facteur de sécurité (FS) défini comme étant le rapport entre la résistance cyclique normalisée du matériau CRR, et la sollicitation cyclique normalisée CSR induite par le séisme à la même profondeur. Pour s’affranchir du risque de liquéfaction, FS doit être supérieur ou égal à 1,3.

\section{Résultats de l'évaluation du risque de liquéfaction}

Cette étude intéresse particulièrement l'emprise des ouvrages de protection où d'épaisses formations de sables et de sables silteux, très sensibles à ce phénomène, ont été repérées. Le calcul de FS au droit de la digue principale montre que celui-ci est inférieur à 1,3 dans les niveaux ci-dessous récapitulés au tableau 1. 
La connaissance de la Mer :

un vecteur du développement durable en Méditerranée

Tableau 1. Profondeur des niveaux à risque à l'emplacement de la digue principale.

\begin{tabular}{|c|c|c|c|c|c|c|c|c|c|}
\hline & \multicolumn{4}{|c|}{ Source proche $M w=4,7$} & \multicolumn{5}{|c|}{ Source lointaine $M w=8,5$} \\
\hline & СРТ21 & CPT22 & CPT24 & CPT25 & CPT21 & СРT22 & CPT24 & CPT25 & СРT27 \\
\hline \multirow{7}{*}{ 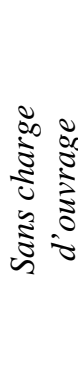 } & $6-6,6$ & $0,4-6$ & $1,2-1,8$ & $0,6-2,8$ & $0,4-0,8$ & $0,4-6,2$ & $0,6-0,8$ & $0,6-2,8$ & $18,6-19,2$ \\
\hline & $7,6-7,8$ & $9,6-10$ & $2,2-2,4$ & & $4-4,2$ & $8,4-8,8$ & $1,2-2,4$ & $6,2-6,6$ & $21,4-21,8$ \\
\hline & & $12-12,2$ & $3,2-5$ & & $5,2-5,4$ & $9,6-10,4$ & $3,2-5,2$ & $13,8-14$ & \\
\hline & & & & & $5,8-6,8$ & $12-12,4$ & & $15,4-16,2$ & \\
\hline & & & & & $7,2-8,8$ & & & $16,6-16,8$ & \\
\hline & & & & & & & & $17,2-17,4$ & \\
\hline & & & & & & & & $18,6-19$ & \\
\hline \multirow{2}{*}{ 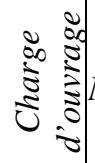 } & & $0,8-3,6$ & $3,2-4,2$ & $0,6-0,8$ & $6-6,6$ & $0,4-6$ & $1,2-2,4$ & $0,6-2,8$ & \\
\hline & Néant & $4,2-4,4$ & $4,6-4,8$ & $1,6-2,2$ & $7,6-7,8$ & $\begin{array}{l}8,6-8,8 \\
12-12,2\end{array}$ & $3,2-5$ & & Néant \\
\hline
\end{tabular}

Dans l'emprise de la digue secondaire (CPT33 et CPT34), tous les coefficients sont supérieurs à 1,3 et par conséquent aucun niveau à risque n’a été repéré.

L'analyse des résultats signale que le séisme lointain est plus favorable au phénomène de liquéfaction que le séisme de source proche.

La charge de l'ouvrage atténue le potentiel de liquéfaction. Seuls subsistent la partie superficielle du sol (environ $3 \mathrm{~m}$ ) et quelques niveaux très réduits en épaisseur.

La zone critique coïncide avec les CPT 22, 24 et 25 sur environ $460 \mathrm{~m}$.

\section{Conclusion}

L’étude géologique et géotechnique du Port Tanger Med met en évidence l'existence de trois vallées traversant le site du port et une dominance des sables et des sables silteux sous les ouvrages de protection susceptibles de liquéfier sous sollicitation sismique. L'analyse du risque, conduite sur la base des CPTU et des séismes de projets, montre que la zone à risque se situe entre les CPT22 et 25 et que le séisme lointain est plus néfaste pour le terrain. La charge appliquée par l'ouvrage réduit ce potentiel de liquéfaction et seule subsiste la partie superficielle du sol d'environ 3m de hauteur.

\section{Références bibliographiques}

CHERKAOUI T.E. (2003). Evaluation de l'aléa sismique : port de TangerMéditerranée. Rapport d'étude.

GEOTER International (2004). Evaluation probabiliste de l'aléa sismique sur le site du port de Tanger Méditerranée. Rapport GTI/TMSA/0105-50.

YOUD T.L., IDRISS I.M. (2001). Liquefaction resistance of soils: summary report from the 1996 NCEER and 1998 NCEER/NSF workshops on evaluation of liquefaction resistance of soils. J. of Geotechnical and Geoenvironnemental Eng., pp 297-313. 